\title{
Imaging in Cor Triatriatum Sinistrum: A Rare Cardiac Entity
}

\author{
Pranav Gupta ${ }^{1}$ Surabhi Kaushik ${ }^{1}$ Sunil K. Puri ${ }^{1}$ \\ ${ }^{1}$ Department of Radiology, Govind Ballabh Pant Institute of \\ Postgraduate Medical Education and Research (GIPMER), New Delhi, \\ India \\ Address for correspondence Pranav Gupta, Department of \\ Radiology, Govind Ballabh Pant Institute of Postgraduate Medical \\ Education and Research (GIPMER), New Delhi, India \\ (e-mail: dr.pranavg@gmail.com).
}

Indian J Radiol Imaging 2021;31:789-790.

We report a case of a 25-year-old, normotensive male patient, who presented with progressive dyspnea (NYHA III). He underwent a battery of tests including transthoracic echocardiography (TTE). which revealed a rare congenital cardiac condition that was later confirmed through a cardiac computed tomography (CT) scan.

The apical four-chamber view on TTE ( - Fig. 1 ) showed a thin horizontal membrane dividing the left atrium into proximal and distal segments with normal heart chambers and atrioventricular $(\mathrm{AV})$ valves. Cardiac CT confirmed the findings with a thin membranous structure running horizontally to bisect the left atrium into a proximal (posterosuperior) and a distal (anteroinferior) chamber (-Fig. 2). A fenestration was seen on the superomedial aspect of the membrane (-Fig. 3 ) through which the two chambers could communicate. The distal segment was in contact with the mitral valve and contained the atrial appendage. The proximal segment received blood from all four pulmonary veins with no anomalous drainage (-Fig. 4). On the basis of these findings, a diagnosis of cor triatriatum sinistrum (CTS) was made.

\section{Discussion}

Cor triatriatum sinistrum is an infrequent cardiac anomaly that accounts for 0.1 to $0.4 \%$ of congenital heart diseases ${ }^{1}$ and was first described by Church in 1868. In this condition, the left atrium is bisected by an anomalous fibromuscular membrane into a proximal chamber or the common embryonic pulmonary vein and a distal chamber or the embryonic left atrium giving rise to the atrial appendage and the AV valve. ${ }^{2}$ The two chambers generally communicate with each other through fenestrations in this inter-atrial membrane. The simplest classification was laid down by Loefller in 1949 , where group one was defined as having no opening, group two as having one or additional small openings, and group three as having a single, large opening. ${ }^{3}$ Group one patients present early in infancy with pulmonary edema and other features of left heart obstruction, while group two and three patients are usually asymptomatic. It is mostly detected incidentally in adult population. Large fenestrations can become obstructed later in life due to fibrosis and calcification, which can then cause symptoms such as dyspnea, orthopnea, and hemoptysis. ${ }^{4}$ Associated cardiac conditions include atrial and ventricular septal defect, anomalous pulmonary vein return, bicuspid aortic and right AV valve, coarctation of aorta, and persistent left superior vena cava. ${ }^{4,5}$

Echocardiography depicts the interatrial membrane beautifully while the fenestrations in the membrane can sometimes be visualized as a jet of increased velocity on color Doppler. CT scan and magnetic resonance imaging (MRI) help in assessing the membrane, cardiac anatomy, and associated congenital anomalies with oblique reformats being extremely useful in visualizing the lesion in entirety. Sometimes, a jet can be seen on CT while MRI can evaluate the flow through the fenestrations and assess other functional parameters including mitral regurgitation.

Treatment in CTS depends on the severity of the patient's symptoms. Incidentally detected lesions in asymptomatic patients do not warrant any active management. Significant dyspnea and pulmonary congestion are treated by diuretics, digoxin, and preload reduction, while surgical intervention is reserved for highly symptomatic patients with significant obstruction. $^{5}$
DOI https://doi.org/ 10.1055/s-0041-1736161. ISSN 0971-3026.
(C) 2021. Indian Radiological Association. All rights reserved.

This is an open access article published by Thieme under the terms of the Creative Commons Attribution-NonDerivative-NonCommercial-License, permitting copying and reproduction so long as the original work is given appropriate credit. Contents may not be used for commercial purposes, or adapted, remixed, transformed or built upon. (https://creativecommons.org/ licenses/by-nc-nd/4.0/)

Thieme Medical and Scientific Publishers Pvt. Ltd., A-12, 2nd Floor, Sector 2, Noida-201301 UP, India 


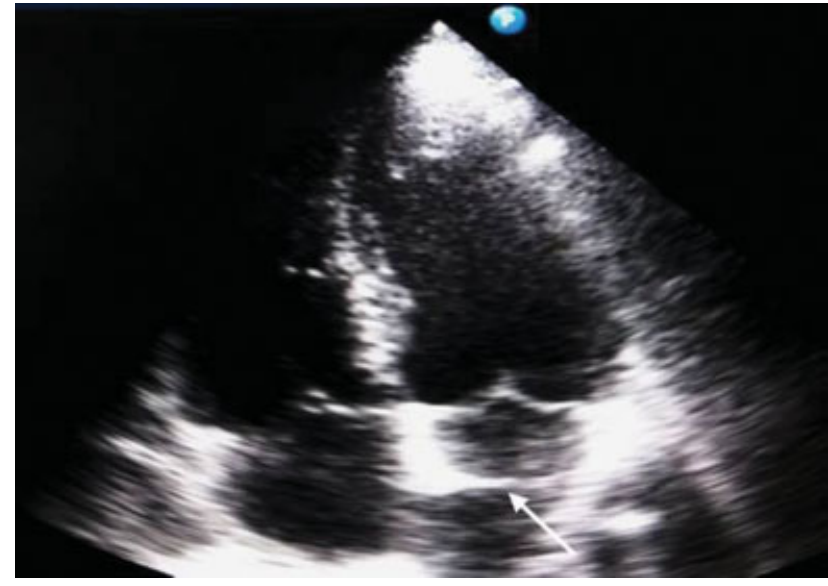

Fig. 1 Four chamber echocardiogram image showing a thin echogenic membrane (straight arrow) dividing the left atrium into proximal and distal chambers.

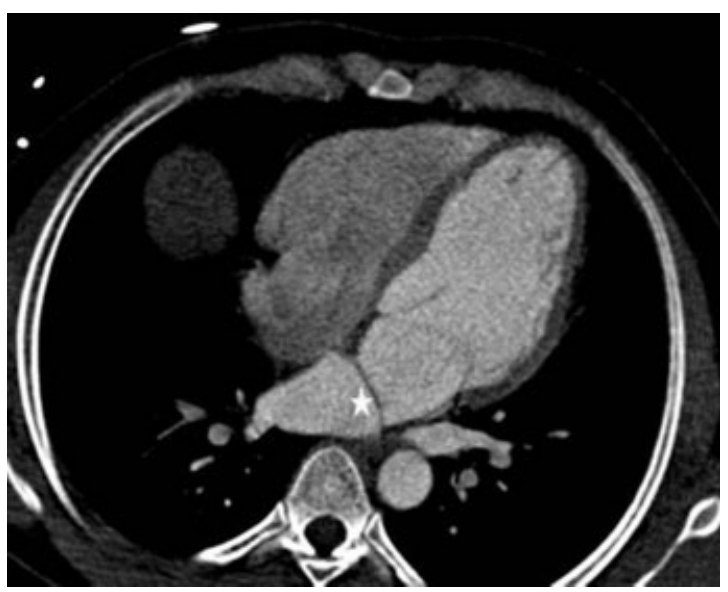

Fig. 2 Axial cardiac CT image revealing the same membrane as a thin hypodense structure (white star) which is seen to bisect the left atrium. CT, computed tomography.

\section{Funding}

None.

Conflicts of Interest

None declared.

\section{References}

1 Bassareo PP, Tumbarello R, Mercuro G. Cor triatriatum and lipomatous hypertrophy of the interatrial septum in the elderly: a case report. Cardiovasc Ultrasound 2010;8:4

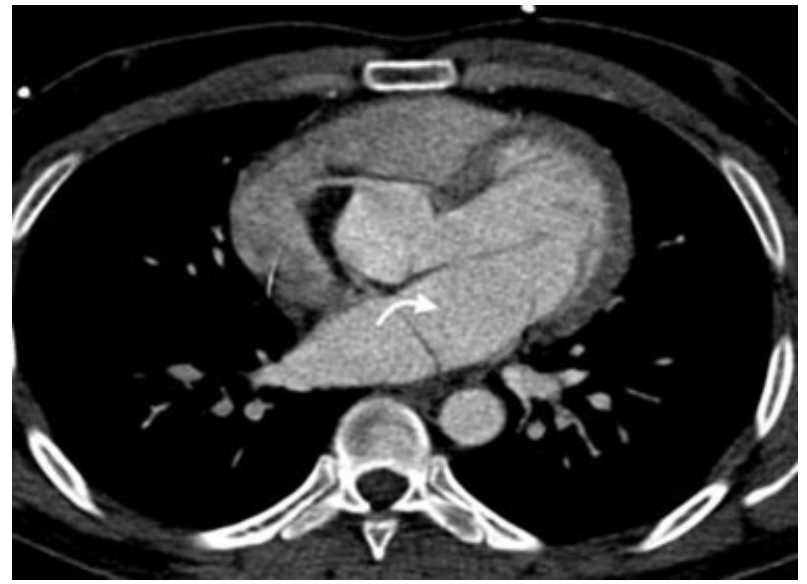

Fig. 3 Superior section on the axial CT images showing a communication between the proximal and distal parts of the left atrium (curved arrow) suggesting a fenestration. CT, computed tomography.

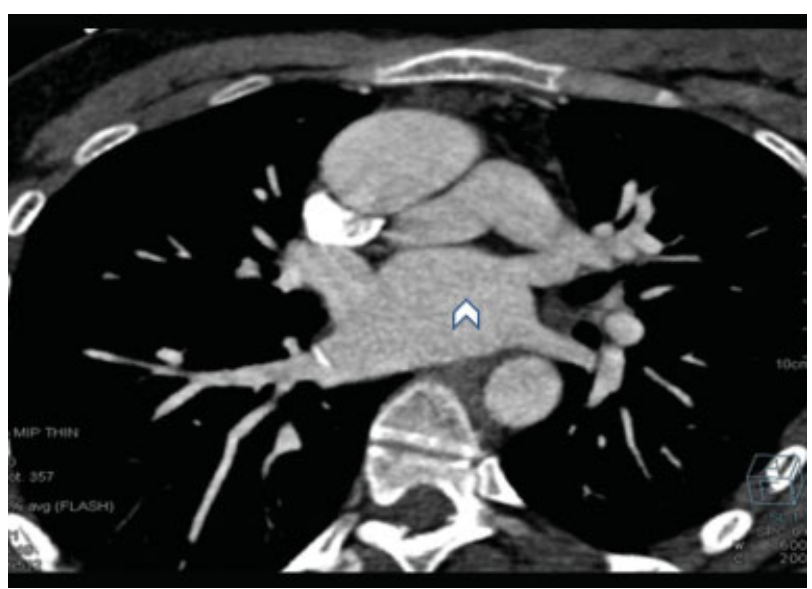

Fig. 4 Axial oblique CT image showing the proximal segment of the left atrium (chevron) receiving blood from all four pulmonary veins. $\mathrm{CT}$, computed tomography.

2 Thakrar A, Shapiro MD, Jassal DS, Neilan TG, King ME, Abbara S. Cor triatriatum: the utility of cardiovascular imaging. Can J Cardiol 2007;23(02):143-145

3 Su CS, Tsai IC, Lin WW, Lee T, Ting CT, Liang KW. Usefulness of multidetector-row computed tomography in evaluating adult cor triatriatum. Tex Heart Inst J 2008;35(03):349-351

4 Zepeda IA, Morcos P, Castellanos LR. Cor triatriatum sinister identified after new onset atrial fibrillation in an elderly man. Case Rep Med 2014;2014:674018

5 Nassar PN, Hamdan RH. Cor triatriatum sinistrum: classification and imaging modalities. Eur J Cardiovasc Med 2011;1(03): $84-87$ 